\title{
Los paisajes del barro
}

\author{
Juan CORTÉs PEDROSA \\ Juan.cortes.pedrosa@gmail.com
}

Recibido: 26 de junio de 2014

Enviado a evaluar: 30 de junio de 2014

Aceptado: 28 de julio de 2014

\section{RESUMEN}

Se propone analizar las características del medio físico y antrópico de la comarca de Tierra de Campos para comprender su paisaje, a través de sus relaciones de causa-efecto. También destaca la importancia del hábitat como elemento clave del territorio y en relación con el resto de elementos, como son la trama parcelaria, los usos del suelo y la red fluvial y de infraestructuras. La singularidad de este entorno no procede solamente de la coherencia de todos sus elementos construidos, sino también del hecho de generar una marcada identidad territorial. Es necesario detectar cuales son las dinámicas socioeconómicas globales que afectan de forma más intensa al paisaje agrario transformando rápidamente el espacio local. Solamente de esta forma se podrán abordar sus consecuencias no deseadas, como la degradación de la imagen física. Se han encontrado dos tipos de impacto visual negativo: la aparición de nuevas construcciones industriales y la decadencia de las edificaciones populares.

Palabras clave: Paisaje, Territorio, Medioambiente, Asentamientos tradicionales.

\section{The landscapes of clay}

\begin{abstract}
The analysis of the physical and human characteristics and the main cause-effect relationships between them is key to understanding the landscape of the region of Tierra de Campos. The importance of the settlement pattern is a key feature of the territory, as well as its relationship with the other elements, like the rural plot types, the land uses and the fluvial and infrastructural network. The singularity of the environment not only stems from the coherence of its built landscape, but also from the fact that it creates an unmistakable territorial identity. It is necessary to find out the global socioeconomic dynamics that are intensely affecting the agrarian landscape, rapidly transforming the local spaces. Only this way will it be possible to handle the undesired consequences, like visual degradation. We have detected two main kinds of negative impact: the emergence of new industrial constructions and the decadence of traditional buildings.
\end{abstract}

Key words: Landscape, Territory, Environment, Traditional settlements. 


\section{Les paysages de l'argile}

\section{RÉSUMÉ}

Il est proposé d'analyser les caractéristiques de la physique et de l'homme à travers la Tierra de Campos à comprendre le paysage, grâce à ses relations de cause à effet. Il souligne également l'importance de l'habitat comme un élément clé du territoire et par rapport aux autres éléments, comme par parcelle, l'utilisation des terres et le réseau de la rivière et de l'infrastructure. Le caractère unique de cet environnement ne vient pas seulement de la cohérence de tous les éléments construits, mais aussi le fait de créer une forte identité régionale. Vous devez identifier quelles sont les dynamiques socioéconomiques globaux qui affectent plus directement le paysage agricole est en train de transformer rapidement l'espace local. Seulement de cette façon peuvent adresser leurs indésirables, comme la dégradation des conséquences physiques d'image. Nous avons trouvé deux types d'impact visuel négatif: l'émergence de nouveaux bâtiments industriels et la baisse des bâtiments populaires.

Mots clés: Paysage, territoire, l'environnement, l'habitat traditionnel.

\section{INTRODUCCIÓN: MARCO TEÓRICO}

Al abordar una investigación sobre el paisaje $\mathrm{e}^{1}$ es necesario precisar la noción que se maneja, teniendo en cuenta que es un término polisémico (Sotelo, J. A., 1991). Enseguida vienen a la mente palabras como entorno, sitio o vista, que están unidas al paisaje, pero no alcanzan a capturar sus múltiples facetas. Desde la disciplina arquitectónica se tiende a considerar el paisaje como un fenómeno predominantemente estético, ligado a su origen pictórico en el contexto cultural europeo (Roger, A., 2007). Esto puede ser consecuencia de la tradicional integración de la enseñanza de la Arquitectura en las escuelas de Bellas Artes. Se trabaja así con una noción de paisaje basada en la concatenación de escenas visuales, dando origen a la disciplina que se ocupa de proyectar los espacios exteriores, denominada apropiadamente como arquitectura del paisaje (Barba, R., 1996).

Sin embargo, este enfoque tiene dos limitaciones que reducen su operatividad en el ámbito teórico. En primer lugar, está el distanciamiento respecto de aquellas ciencias, como la geografía y la ecología, que ofrecen perspectivas de gran interés para el debate actual sobre el medio ambiente y el patrimonio construido. Entre ellas cabe destacar la ecología del paisaje, que estudia las pautas morfológicas que se repiten en el espacio, relacionándolas con fenómenos como la biodiversidad, los flujos energéticos o la intervención humana en el entorno natural, analizada a

${ }^{1}$ El presente artículo se basa en una ponencia del autor con el mismo título, presentada en el X'I Congreso Internacional de Arquitectura en Tierra, celebrado en Valladolid en septiembre de 2014. 
través del tapiz de usos del suelo. En segundo lugar, se corre el riesgo de contemplar el paisaje como un ente estático, congelado en el tiempo. De esta forma, se estaría obviando su dimensión temporal, cuando esta evolución es de suma importancia para comprender una realidad rápidamente cambiante. Esto evitaría grandes inversiones y esfuerzos encaminados hacia una conservación a ultranza de la fisionomía territorial, con enfoques discutibles y resultados frecuentemente estériles. Si no se logran identificar los procesos subyacentes que modifican constantemente el entorno, ya sean biológicos o culturales, no se podrán gestionar los paisajes rurales de forma eficaz y mantener su importancia en el futuro (Diaz Pineda, F., 2003).

Para analizar este aspecto diacrónico es de gran utilidad asimilar el paisaje a un sistema (Sauer, C., 1925), es decir, un conjunto de elementos relacionados entre sí por un número restringido de interacciones. Estos elementos se pueden agrupar en tres categorías básicas, desde un punto de vista morfológico: superficies, líneas y puntos (Forman, R. y Godron, M., 1986). Estas categorías van unidas, a su vez, a las funciones que desempeñan. Por ejemplo, el primer grupo lo forman las mallas y superficies que ocupan una porción extensa del territorio, como la trama del parcelario rural. Estos elementos representan el principal uso del suelo y su importancia ecológica es grande, al albergar la mayoría de procesos naturales (Forman, R., 1995). Los componentes lineales suelen servir para el traslado de materia y energía dentro del sistema, o bien forman los límites que sectorizan el espacio y crean ámbitos diferenciados en sus márgenes. Los elementos puntuales carecen de dimensión aparente al nivel territorial y controlan los principales flujos ecológicos y socioeconómicos (Raffestin, C., 1980). En el ámbito rural aparecen en forma de asentamientos o concentraciones industriales. Una vez definidos estos elementos se estudian las restricciones de sus relaciones mutuas, obteniéndose así un cierto grado de previsibilidad (Margalef, R., 1980). Esto sirve para realizar un diagnóstico de la situación actual y proponer, en su caso, medidas correctoras efectivas. En resumen, el presente estudio parte de un enfoque integrador y sistémico para establecer las relaciones de causa-efecto entre las dinámicas territoriales y su configuración visual. Con este propósito, se adoptan los tres niveles de análisis del paisaje, que propone el geógrafo Eduardo Martínez de Pisón (2004):

a) Procesos no-visibles que tienen lugar en el territorio (ecológicos y socioeconómicos)

b) Morfología o materialización de estos procesos sobre la base físico-natural

c) Percepción de estas relaciones espaciales por parte del ser humano 


\section{EL SOPORTE TERRITORIAL}

Este trabajo se centra en la comarca vallisoletana de Tierra de Campos, que ocupa la parte norte de la provincia y se considera como un ámbito propio de ordenación territorial (Santos y Ganges, L. y Peiret i Carrera, A., 2001). Su posición geográfica lejana de las cadenas montañosas de Castilla y León, no propicia grandes contrastes físico-ambientales, constituyendo una unidad paisajística (García Merino, L. V., 1988). Tradicionalmente se consideró que este territorio formaba parte de una "región natural" del mismo nombre y mayor superficie que se extendía hacia las provincias vecinas. Sus peculiares características ecológicas y socioeconómicas le dotaron de una identidad propia dentro del contexto regional. Sin embargo, la división provincial creada impidió que esta entidad tuviera competencias políticas y administrativas, generándose progresivamente cierta diferenciación entre sus partes.

\subsection{EL MEDIO FÍSICO}

Desde un punto de vista físico-ambiental destacan dos unidades geomorfológicas, la campiña, que ocupa la mayor parte de la superficie, y el páramo compartido con la comarca de Montes Torozos. Estos páramos son plataformas techadas por una cobertura de calizas pontienses que se elevan unos cien metros por encima de las campiñas, llanuras modeladas en materiales blandos, poco contrastadas topográficamente y debidas sobre todo al efecto de la erosión. El conjunto es bastante uniforme y los únicos accidentes del relieve que interrumpen la campiña son algunos cerros testigos y colinas con las cumbres aplanadas, llamadas tesos. La horizontalidad del espacio define un paisaje homogéneo, favoreciendo un hábitat que ocupa el territorio de forma regular. Esta uniformidad del medio influye también en la aparición de un modelo típico de núcleo de población, que responde a las necesidades políticas y socioeconómicas antes que a la fisionomía del terreno. La geomorfología de la comarca es propicia para la agricultura y se puede afirmar que favorece la aparición de un paisaje agrario de campos abiertos con unos asentamientos que forman una malla casi isótropa, maximizando así la explotación del territorio (Benito Martín, F., 2000).

La orografía no se ve demasiado influida por la red fluvial existente (formada por los ríos Cea, Valderaduey, Ahogaborricos y Sequillo), debido a la suavidad de sus interfluvios. Exceptuando el Cea, se trata de cauces de muy escaso caudal que atraviesan la zona de noreste a suroeste. La falta de obstáculos de las subcuencas y la tranquilidad de los cursos favorecen la labor agrícola y la movilidad dentro de la comarca. Existe otro factor que también ejerce una fuerte influencia paisajística: los suelos aluviales de las inmediaciones de los ríos, ricos y húmedos, que junto con la mayor altura de la capa freática, permiten una vegetación frondosa y la explotación de pequeños huertos. Dentro de la red hidrográfica destaca el Canal de Castilla, una de las obras nacionales de ingeniería hidráulica más importantes entre los siglos XVIII y XIX, que crea un llamativo corredor verde a su paso. 
Figura 1. Vista panorámica de Tierra de Campos desde el páramo hacia la campiña.

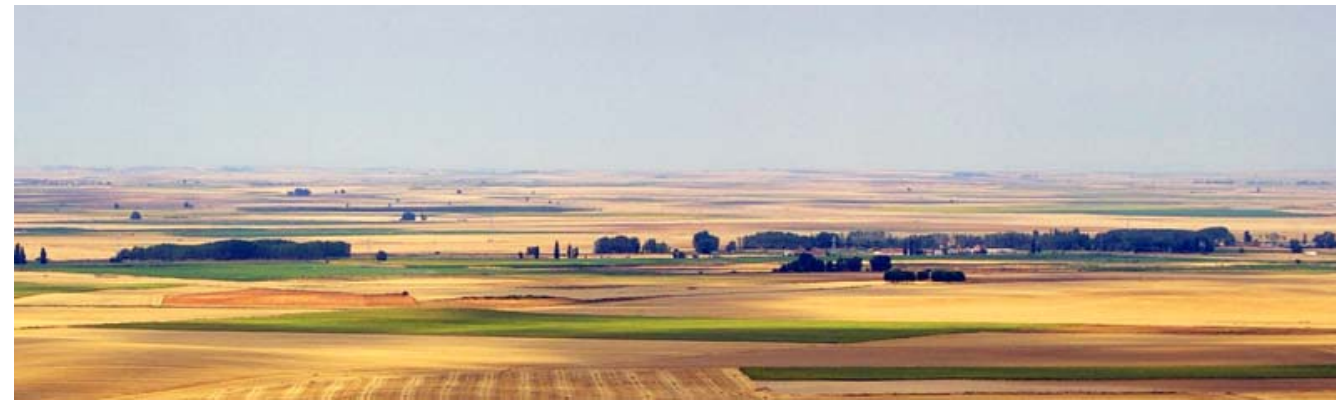

Fuente: Elaboración propia

Desde el punto de vista litológico predominan los materiales de tipo arcilloso, de color amarillento-rojizo, debido a los óxidos liberados por los materiales de base (García Merino, L. V., 1988). Por su gran soltura resultan aptos para el cultivo y han favorecido la labor agrícola desde hace, al menos, veintidós siglos. Otro hecho fundamental es la ausencia de suelos pétreos, lo cual creaba unas restricciones para la construcción en las etapas preindustriales, imponiéndose el "barro" como material por excelencia. Esto generó una severa uniformidad visual en las edificaciones de la comarca, con la excepción de los elementos representativos como iglesias o castillos, lo que supone uno de los principales valores estéticos del paisaje (Flores López, C., 1974; Benito Martín, F., 1998; Cortés Pedrosa, J., 2013).

El clima de Tierra de Campos es de tipo mediterráneo continental con un largo y frío invierno y unos veranos secos y cortos, que destacan por unas elevadas temperaturas diurnas. Estas altas máximas unidas a la bajada nocturna, debido a la irradiación y la altitud, provocan una oscilación térmica de unos 16 grados. Esto tiene unas fuertes repercusiones sobre la arquitectura tradicional, como la necesidad de construir muros de gran espesor para hacer frente a estas variaciones mediante la inercia térmica. Los conjuntos edificados presentan, por lo tanto, una considerable masividad y ausencia de grandes huecos. Las precipitaciones son moderadas por la alta frecuencia de cielos despejados, pero irregulares a lo largo del año, concentrándose en las estaciones de otoño y primavera. Esto permitió la construcción de cubiertas con poca inclinación, que acentúan la extrema horizontalidad del paisaje.

Otra singularidad de la comarca es la práctica inexistencia de vegetación arbórea, exceptuando algunas manchas de monte de encinas y robles en los límites con los páramos. Los intentos de repoblación en el siglo XX se concentraron en las cuestas de transición con la campiña, pero no tuvieron éxito debido al escaso desarrollo de los pinos. Únicamente la presencia de los ríos y canales conforma líneas verdes de arbolado y concentra los escasos cultivos de regadío. El largo e intenso proceso de explotación antrópica del suelo, unido a las características de la campiña, generó el desnudo paisaje actual (Baraja Rodríguez, E., 2012). Esta situación limitó también el uso de elementos leñosos en la construcción tradicional, exceptuando la viguería de 
las cubiertas y los forjados. Por lo tanto, el sistema constructivo más extendido es el muro de carga macizo, en forma de tapial o aparejo de adobe, ya que la escasez de leña limitaba la fabricación de ladrillo (Sáinz Guerra, J.L. et alii, 2012).

\subsection{EL MEDIO HUMANO}

El más antiguo testimonio humano en el territorio procede de los vacceos, pueblo prerrománico que practicaba una agricultura colectivista y una ganadería trashumante. Estas dos actividades económicas primordiales han pervivido hasta hoy, dando lugar a la cultura propia de la comarca. A nivel espacial existía ya una estructura descentralizada basada en los oppida, pequeños núcleos fortificados que articulaban el territorio y organizaban la economía local (Hernández García, R., 2011). Se suceden después las etapas de dominación romana y visigótica, que apenas alteran este sistema de asentamientos. Pero esta situación termina con la entrada de los musulmanes en la Península Ibérica, pasando a ser un territorio fronterizo casi despoblado entre los cristianos y los árabes. Las incursiones militares y las migraciones afectan a los núcleos, que pierden poco a poco su población y no es hasta el siglo IX cuando la comarca se repuebla durante la primera fase de la Reconquista, originándose muchos de los núcleos actuales. En algunos casos se aprovechaba el primitivo emplazamiento defensivo, llamado castro, mientras que en otros se fundaban nuevas villas fronterizas por parte de los reinos de León y de Castilla (Benito Martín, F., 2000). Según relata Justo González Garrido (1941), estos asentamientos se desarrollaron inicialmente "por medio de pequeñas explotaciones rurales, que a veces señoreaban algunas aglomeraciones urbanas". En este momento clave, la Baja Edad Media, se estructura definitivamente el territorio con tres características: un gran número de habitantes por núcleo, una baja densidad de población y un hábitat concentrado (Vaca Lorenzo, A., 1977). Si en un primer momento el poblamiento tiene la función estratégica de consolidar el dominio político, tampoco se debe olvidar que esta malla isótropa permitió un desarrollo económico ligado a la agricultura extensiva. Las oleadas de crecimiento demográfico causaron una gradual extensión de la superficie cultivada, que no se extendió hacia los páramos hasta el siglo XIX, mediante el proceso desamortizador. Esta progresiva "estepización" provoca que sean los objetos artificiales, sobre todo los núcleos de población y las construcciones auxiliares, como palomares o cobertizos, los que caracterizan visualmente el espacio, en ausencia de elementos naturales predominantes.

Durante el siglo XX tiene lugar una transición desde una economía agraria de subsistencia, intensa en mano de obra, hacia un modelo productivista que se caracteriza por la alta mecanización de las labores. Esto causa la migración de una parte considerable de la población a los pujantes centros urbanos, como la ciudad de Valladolid, donde se concentra la actividad industrial y terciaria. Tierra de Campos se especializa en un modelo agrícola cerealista, que depende hoy de la Política Agraria Común de la Unión Europea. También existe una importante cabaña ovina que genera una incipiente industria de transformación de productos alimenticios. Todos estos cambios estructurales sufridos por la agricultura y la ganadería de la región, como por 
ejemplo la estabulización del ganado, han tenido como efecto la aparición de numerosas construcciones industriales salpicadas por el paisaje. No guardando ninguna relación con las características de la arquitectura popular, generan un impacto negativo en el entorno rural (Cortés Pedrosa, J., 2014).

A nivel demográfico la comarca cuenta, a día de hoy, con una población total de 17.869 habitantes, 9.236 hombres y 8.632 mujeres (INE, 2013). Esto supone un desequilibrio notable por sexos, atribuible a la falta de empleo en otros sectores distintos de la agricultura, a pesar de los intentos de diversificación emprendidos por las administraciones públicas. Otra característica es el envejecimiento, aproximadamente un $40 \%$ de la población es mayor de 65 años. La tendencia poblacional descendente a partir de la mitad del pasado siglo se interrumpe solamente con un pequeño crecimiento entre los años 2001 y 2005, debido a la llegada de emigrantes extranjeros por el auge de la construcción de segundas viviendas (Aparicio Gervás, J. M. et alii, 2005). El principal problema social es, por tanto, el proceso de pérdida de población (aproximadamente un 50\% en los últimos 60 años), que junto con el envejecimiento y la masculinización hacen peligrar el futuro de los pueblos más pequeños (Martínez Fernández, L. C. y Delgado Urrecho, J. M., 2013). Estos hechos implican también la progresiva decadencia del patrimonio rural por la falta de uso y mantenimiento. No existen apenas núcleos importantes, de hecho Medina de Rioseco, el mayor municipio, no supera los 5.000 habitantes. Los dos siguientes en dimensiones, Villalón de Campos y Mayorga, tienen menos de 2.000 pobladores y no es previsible un aumento a corto o medio plazo, debido a los problemas estructurales existentes. La mayoría de asentamientos, de hecho, no superan los 500 habitantes y su densidad de población es inferior a 10 habitantes por $\mathrm{km}^{2}$.

\section{LA FORMA DEL PAISAJE}

\subsection{LOS CULTIVOS Y LA TRAMA PARCELARIA}

La presión antrópica en la comarca produjo una gradual sustitución de los ecosistemas primigenios de bosques por llanuras herbáceas. Nos encontramos actualmente con un monocultivo de secano, que abarca la casi totalidad de la superficie hasta sobrepasar el 85\% de ocupación del suelo, según las estimaciones de Eugenio Baraja Rodríguez (2012). Aunque el trigo sigue siendo el principal cultivo, aumentan los cereales forrajeros, como la cebada, destinada a las numerosas explotaciones ganaderas. Aparecen también las leguminosas, el maíz y el girasol, aunque el regadío ha tenido escaso éxito, a pesar de los intentos políticos como el Plan de Tierra de Campos de 1965. El viñedo desapareció prácticamente a finales del siglo XIX debido a la plaga de la filoxera y no ha vuelto a implantarse, por lo cual no existen apenas parcelas con vegetación leñosa que aporten diferentes texturas al paisaje. 
Figura 2. Trama parcelaria del municipio de Cabreros del Monte en 1956 (izda.) y 1977.
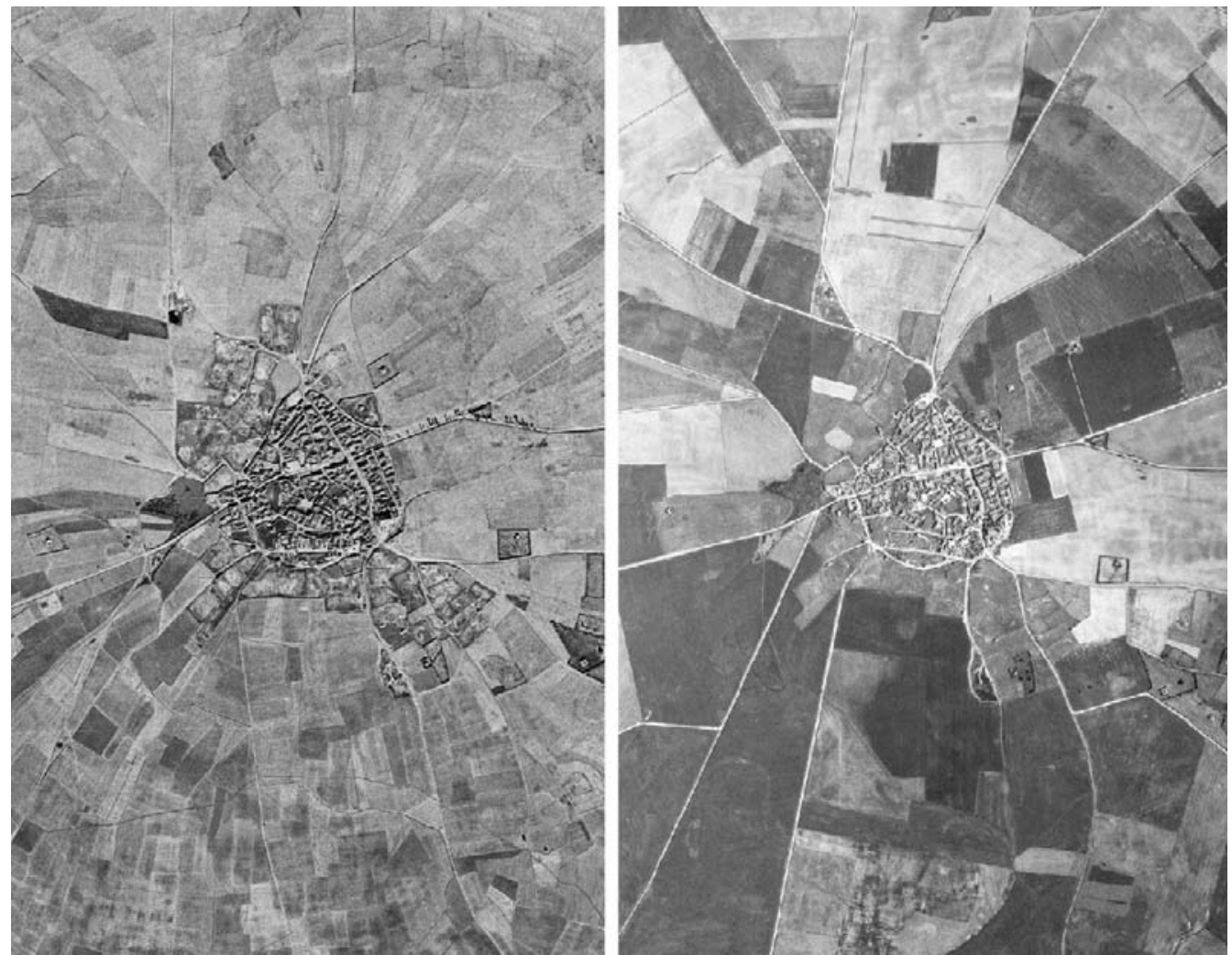

Fuente: Fotografías aéreas del Instituto Geográfico Nacional.

Existía tradicionalmente una trama parcelaria muy fragmentada debido a las "reparticiones" después de las herencias, componiendo un tapiz irregular y diverso. Esto se modifica con la reforma de la Concentración Parcelaria, iniciada a mediados del siglo XX, que impulsa un fuerte proceso de concentración de la propiedad, orientado a optimizar y aumentar la producción agrícola mediante la mecanización de las labores. Se pasa así de un tipo de propiedad disperso entre varios municipios y con unidades de entre 4 y $20 \mathrm{Ha}$ a una situación altamente funcional y dominada por las explotaciones familiares con parcelas de entre 120 y $300 \mathrm{Ha}$ (Baraja Rodríguez, E., 2012). Esta mayor regularidad también ha tenido consecuencias sobre el paisaje, que se ha vuelto más homogéneo y ha perdido riqueza geométrica, predominando actualmente las formas rectangulares simples y desapareciendo numerosos hitos singulares. Sin embargo, estos procesos no han causado un gran impacto a nivel perceptivo, debido a la horizontalidad del espacio que atenúa los cambios de trazado en el plano del suelo, teniendo en cuenta la ausencia de elementos tridimensionales, como cercas, muretes o aterrazamientos. 


\subsection{LAS REDES VIARIAS Y FLUVIALES}

A pesar de su ubicación en el centro de la región castellanoleonesa, la comarca no está dotada de una estructura viaria de primer nivel, debido quizás a su débil demografía y escaso peso económico. La única vía de importancia nacional existente es la autovía A-6 que roza su límite occidental. Si a esto añadimos la ausencia de grandes infraestructuras ferroviarias, hídricas (a excepción del Canal de Castilla) o energéticas, se puede afirmar que no existen elementos lineales que generen un gran impacto visual o ecológico. El eje vial que vertebra el territorio de Norte a Sur es la N-601 entre Valladolid y León, pasando por los núcleos de Medina de Rioseco y Mayorga. Otra vía de importancia es la N-610 entre Palencia y Benavente, eje transversal en dirección Este-Oeste. Existe además una red local de carreteras provinciales para el tráfico interior, siguiendo un esquema radial que tiene como centro a Medina de Rioseco. A nivel municipal destaca la red de caminos rurales, en cuanto a su integración con el trazado viario de los núcleos, facilitando el acceso al terrazgo. Esta malla crea una intensa relación visual entre el núcleo y su entorno, en forma de plazas o ensanchamientos en los encuentros entre ambos.

Figura 3. Esquemas de red viaria (izda.) y fluvial en la comarca de Tierra de Campos.
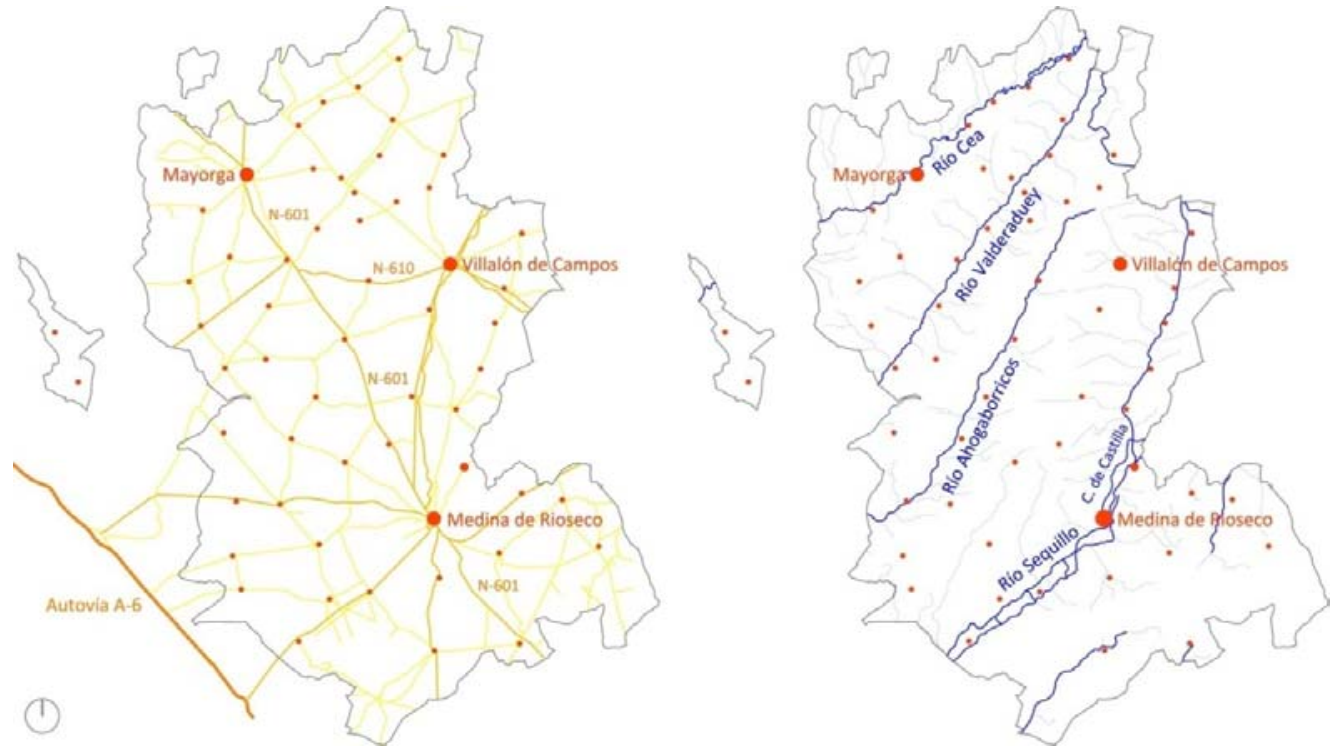

Fuente: Elaboración propia, a partir de la cartografía del Instituto Geográfico Nacional (sin escala).

En cuanto a los ríos, su modesta anchura y escaso caudal limitan su importancia dentro del paisaje. Sin embargo, su presencia fue un factor decisivo para el emplazamiento de los núcleos por su condición de barreras defensivas, definiendo 
unos vectores en dirección SO-NE. A pesar de la fuerte atracción que generan los ríos, debido a la fertilidad de sus suelos y al suministro de agua, no se llegan a integrar apenas en el trazado urbano. Esto se debe a la insalubridad de las vegas durante la sequía veraniega y a las crecidas, causadas por la escasa pendiente y poca profundidad de sus cauces.

\subsection{LA MALLA DE ASENTAMIENTOS}

El poblamiento forma una malla altamente regular, favorecida por la uniformidad geográfica. Las condiciones típicas del hábitat son una distancia media entre núcleos de entre 5 y $10 \mathrm{~km}$ y la existencia de un terrazgo con una superficie aproximada de 25 o $30 \mathrm{~km}^{2}$ por cada pueblo, situado de forma central respecto del mismo (Benito Martín, F. 2000). Teniendo en cuenta que el número de habitantes por núcleo no superaba generalmente los 500, se estima que el ratio de población por recursos ecológicos disponibles era el apropiado para su subsistencia. Los pueblos más importantes de la comarca son Medina de Rioseco, Villalón de Campos y Mayorga, que se mantienen como los centros económicos y sociales, proveyendo al resto del territorio de los servicios esenciales. El resto de núcleos son eminentemente rurales y de pequeño tamaño, pero cumplen con su función de no dejar vacíos en el sistema territorial.

Los núcleos reflejan aún el predominio de un trazado típico, que se caracteriza por una forma altamente compacta. Esto es consecuencia del origen histórico del sistema de asentamientos, que requería de una red de núcleos amurallados para consolidar el dominio militar del territorio. La morfología urbana, nítida y fácilmente legible, se debe al trazado de las antiguas murallas o cercas, que se mantiene gracias a las vías de ronda perimetrales que ocupan su mismo lugar, organizando el acceso a las calles interiores. Esta característica es de gran importancia para el paisaje, ya que los pueblos presentaban una imagen perceptiva unitaria, reforzada por la homogeneidad tipológica y constructiva de las edificaciones. Sin embargo, la forma circular ideal, generada por anillos concéntricos alrededor de la iglesia o el castillo, no aparece casi nunca debido a factores secundarios que deforman la trama. En primer lugar está la topografía, que obliga a una adaptación del tejido a los cerros, tesos o laderas pronunciadas. En segundo lugar, la presencia de un elemento lineal como un río o un camino puede crear un vector de crecimiento a su paso, "estirando" de esta forma el asentamiento. La figura orgánica resultante recuerda vagamente en muchos casos a una alubia o un riñon (Moya, L. et alii, 2014). La casi total ausencia de otras referencias naturales, como accidentes topográficos, grandes cauces fluviales o extensos espacios arbolados, permite afirmar que los núcleos ejercen un papel decisivo a la hora de estructurar y jerarquizar visualmente el territorio. Por ello, se ha estudiado la evolución morfológica de los asentamientos, con el objetivo de identificar las transformaciones que se han producido a partir de la industrialización iniciada en el siglo XX. 
Figura 4. Núcleos de población de Tierra de Campos, según datos de 2013. Escala 1:300.000.

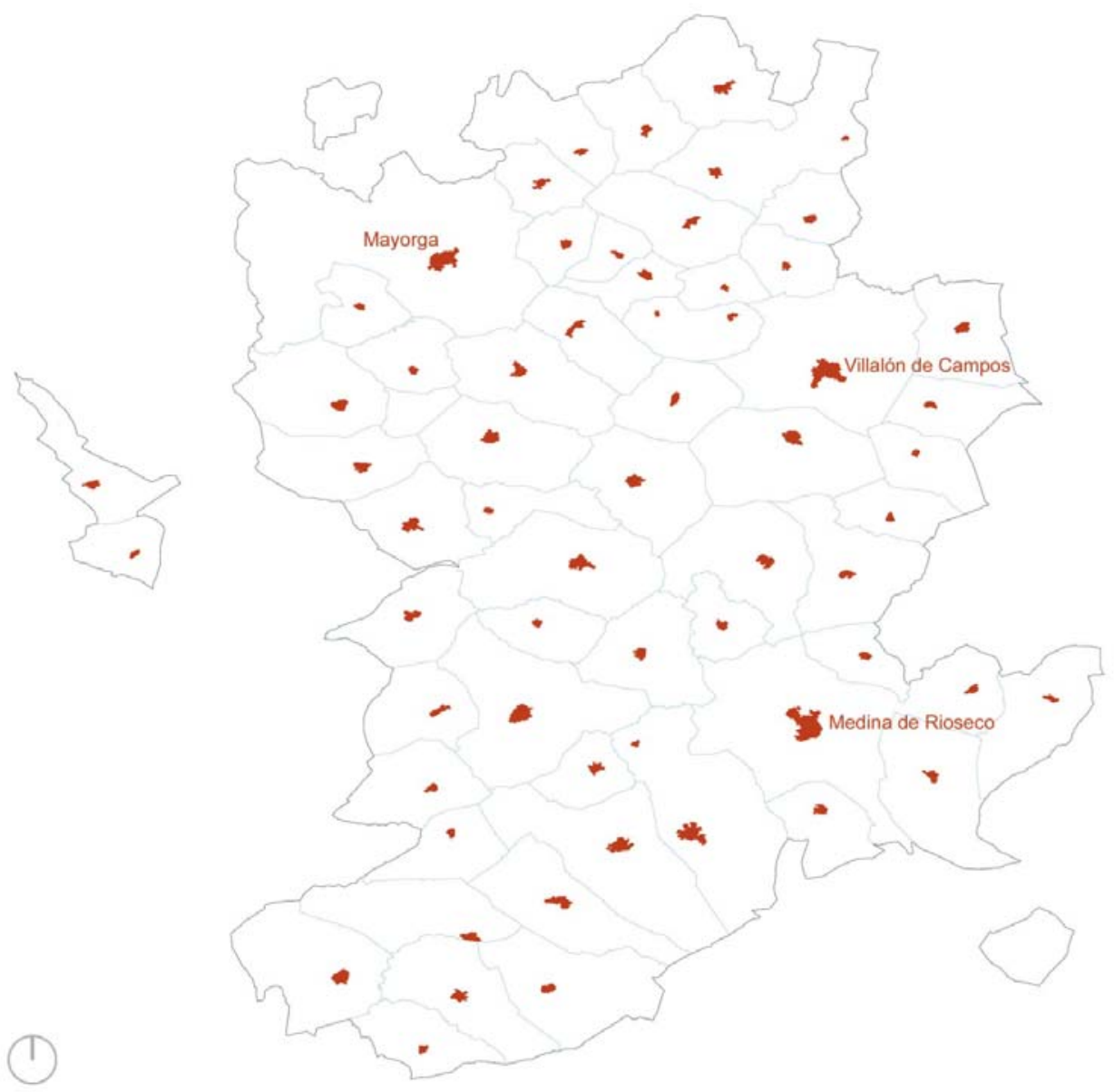

Fuente: Elaboración propia, a partir de fotografías aéreas del Instituto Geográfico Nacional.

El caso de Medina de Rioseco muestra algunos de los procesos característicos que afectan a los asentamientos de Tierra de Campos. La documentación histórica ${ }^{2}$

${ }^{2}$ Según el "Mapa de Medina de Rioseco, 1852, por Francisco Coello" de Francisco Coello. Extraído y montado del mapa general de Valladolid, año 1852, de Francisco Coello. Fuente: http://commons.wikimedia.org/wiki/File:Mapa_de_Medina_de_Rioseco,_1852,por_Francisco Coello_(assembled).jpg\#mediaviewer/File:Mapa_de_Medina_de_Rioseco,_1852,_por_Franc isco_Coello_(assembled).jpg 
muestra un núcleo típicamente compacto, que se encuentra encajado entre el cauce del Río Sequillo y el embarcadero del Canal de Campos, ramal del Canal de Castilla. Su morfología es nítida, gracias al trazado aún perceptible de la antigua muralla y el tejido edificatorio es relativamente homogéneo, en cuanto a su escala y textura. El desarrollo industrial de principios del siglo XX provoca la aparición de una línea ferroviaria, que crea un nuevo límite para el crecimiento urbano, contribuyendo mantenerse así su forma. Pero la nueva infraestructura resta actividad económica al Canal de Campos, que se degrada progresivamente por el desuso, hasta la recuperación efectuada a finales del siglo pasado cuando se restaura el embarcadero y los pabellones laterales que servían para el almacenamiento del cereal transportado en barco.

Figura 5. Aumento de superficie urbana de Medina de Rioseco entre 1956, 1977 y 2013.

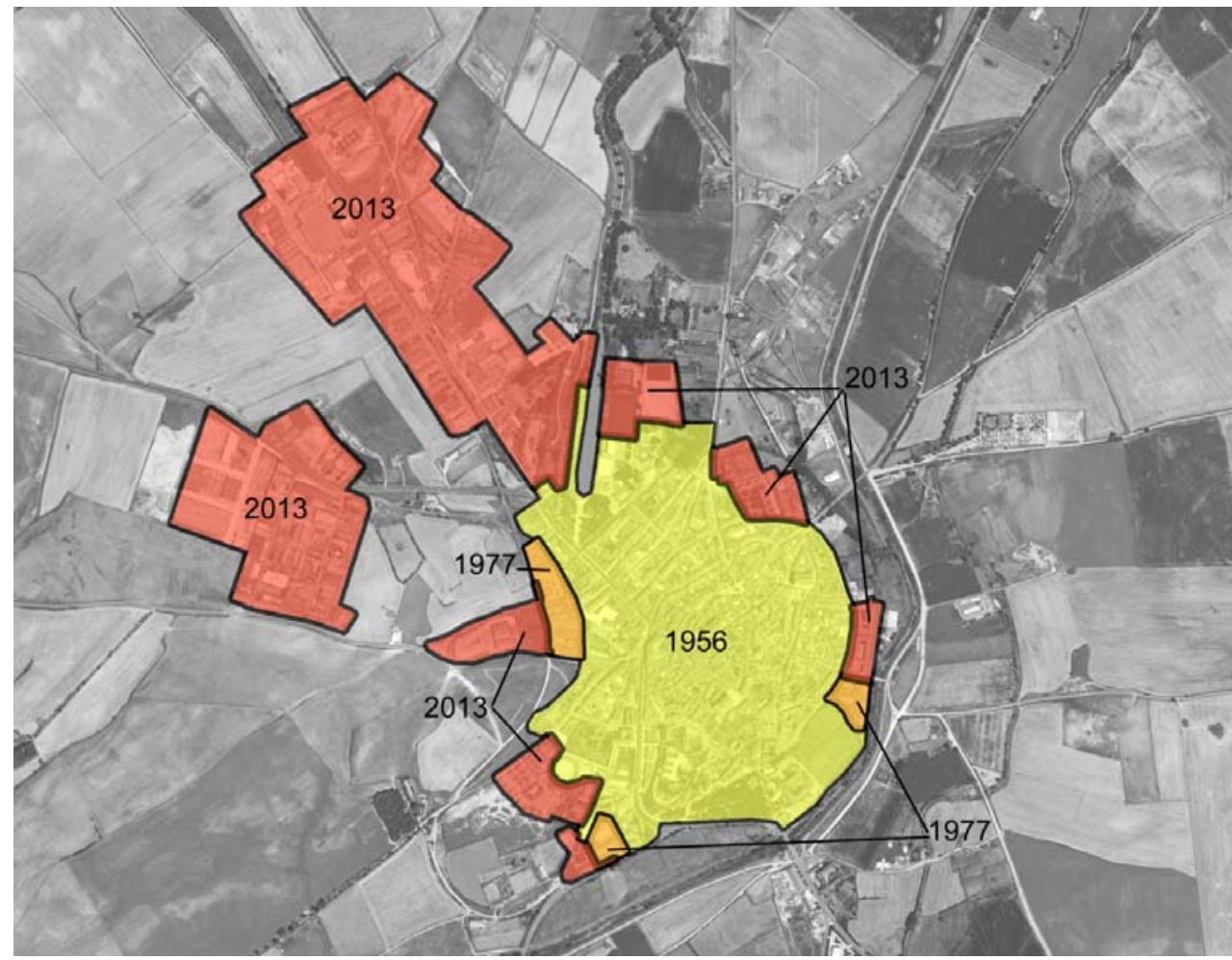

Fuente: Elaboración propia, a partir de fotografías aéreas del Instituto Geográfico Nacional.

Si bien la población del municipio crece en unos 250 habitantes entre 1900 y 1950 , explicando el aumento de superficie urbana, no se altera la percepción global del núcleo al producirse la extensión en forma de "mancha de aceite". Por el contrario, en la ortofoto más reciente se aprecian dos nuevos desarrollos industriales concentrados 
en la parte occidental del asentamiento, debido a las barreras ambientales presentes en el resto del perímetro. Estas nuevas áreas contrastan con el núcleo histórico por su crecimiento axial, en paralelo a la red viaria. En uno de los casos, el ensanche industrial se extiende linealmente en dirección noroeste a lo largo de la N 601, principal vía de acceso a la localidad, distorsionando la secuencia de aproximación al núcleo. Además de esto, su parcelación ortogonal y la escala de sus nuevas edificaciones, grandes naves industriales y edificios del sector terciario, no son coherentes con las características típicas del asentamiento. Esto genera una alteración significativa de la morfología tradicional, que pierde compacidad y legibilidad.

Además de los núcleos existen otros hitos que conforman el paisaje: las edificaciones agrarias, como palomares, casetas y chozos de pastor. Su riqueza estética, generando en ocasiones formas orgánicas, y el contraste con la austeridad del tejido edificatorio residencial, aporta un contrapunto visual que enriquece el entorno. Resulta especialmente sorprendente la variedad de tipos de palomar, existiendo varias hipótesis que explican su exuberancia formal (Roldán Morales, F. P., 1983). Los palomares se encuentran distribuidos por toda la comarca, ya sea de forma aislada o formando conjuntos, y en ocasiones se ven cercados por una tapia, que delimita un pequeño recinto. Generalmente, no aparecen demasiado alejados de los núcleos de población por motivos de conveniencia y para poder ejercer cierto control sobre su acceso. Los tipos más habituales son los redondos y cuadrados, aunque en ocasiones se encuentran también palomares rectangulares, poligonales o mixtos. Actualmente, muchos de ellos están en grave peligro de ruina al caer en desuso por el cambio en el estilo de vida de los habitantes de la comarca.

Figura 6. Palomares redondos y poligonales en Barcial de la Loma.

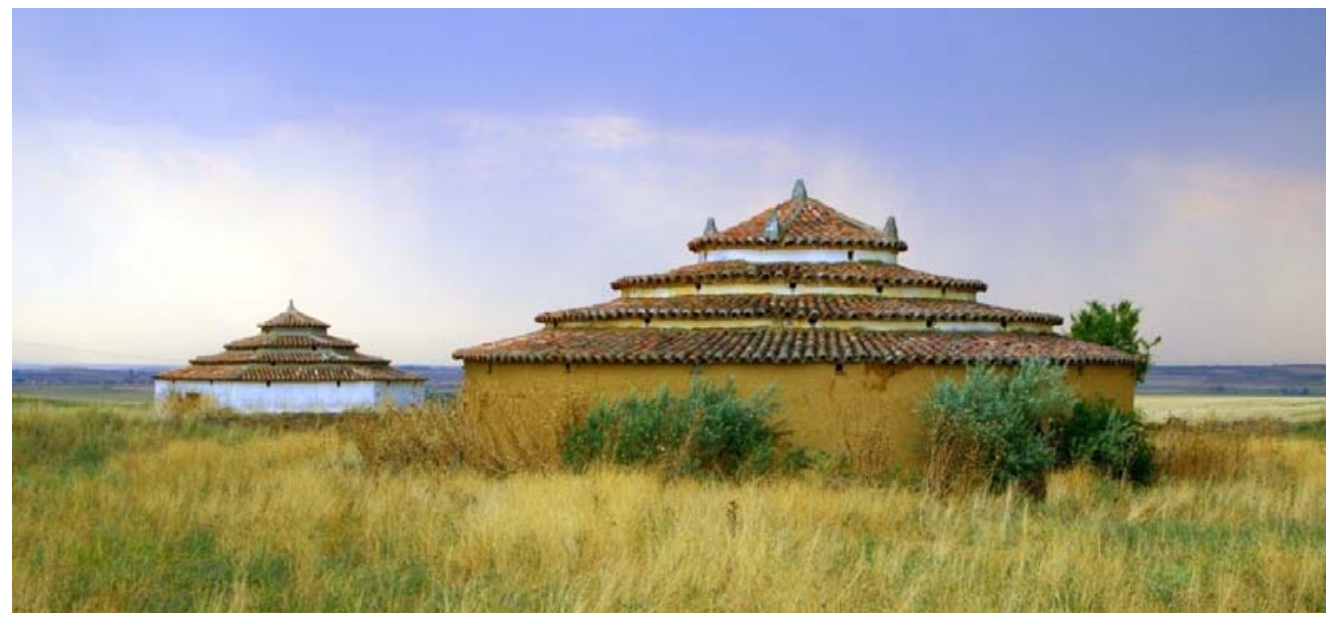

Fuente: Elaboración propia. 


\section{LA CALIDAD ESCÉNICA}

La elección del emplazamiento define la primera relación perceptiva del asentamiento con el terreno. La mayoría de núcleos tienen su origen en una fortificación defensiva situada en una elevación cercana a un cauce, cerca de alguno de sus vados. Posteriormente se suele producir un crecimiento urbano en dirección hacia la llanura, para extender su superficie. Las necesidades estratégicas aconsejaban afianzar una posición elevada para controlar visualmente el entorno. Por este motivo, se favorecía también el asentamiento en las laderas de los cerros o en los escalones del páramo, que permitían además cierto resguardo frente al viento y el aprovechamiento de las fuentes naturales que brotaban en estos puntos (Roldán Morales, F. P., 1996).

La mayoría de los núcleos repite un mismo esquema tridimensional, que se puede denominar como piramidal. Esto es debido a la progresiva ganancia en altura desde el perímetro, compuesto por tapias bajas que cierran corrales y construcciones agregadas, hasta el centro del pueblo, ocupado por una o varias iglesias sus respectivas torres, pasando por el caserío intermedio de una o dos plantas. Esta volumetría permite una óptima integración visual en el entorno por el gradiente de alturas desde el terrazgo hasta el centro del asentamiento. La unidad cromática, gracias al uso de los materiales locales, como el adobe, el tapial y la teja árabe, refuerza esta característica.

Figura 7. Vista del núcleo de población de Herrín de Campos.

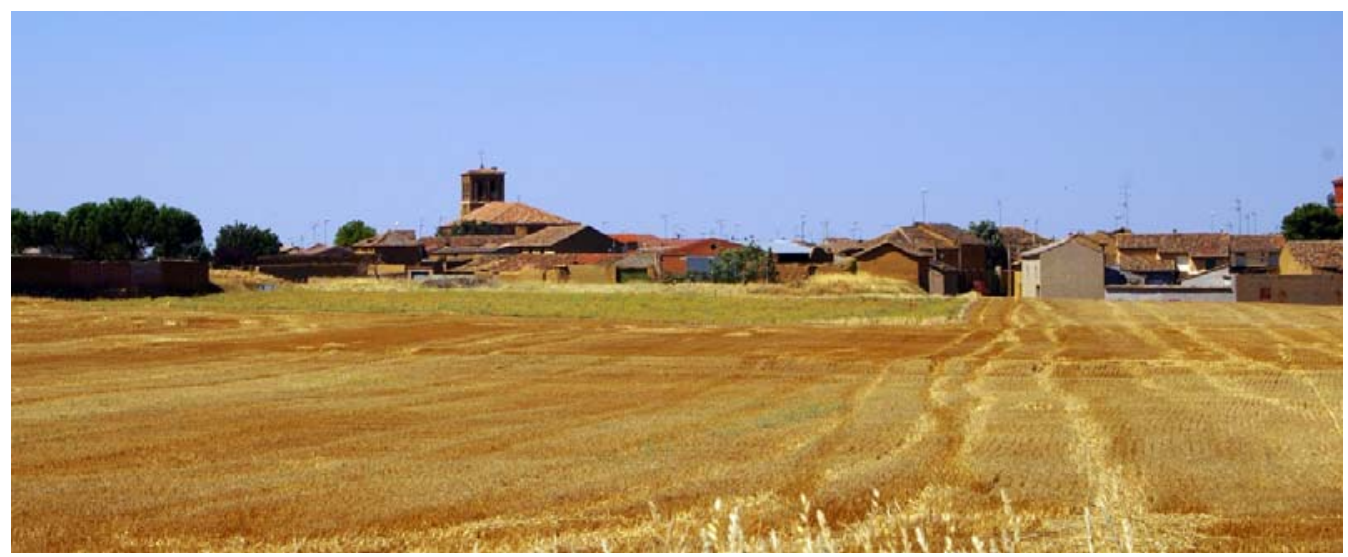

Fuente: Elaboración propia.

Todo ello permite percibir el núcleo como un elemento más del medio, siendo éste uno de los mayores atractivos visuales para el observador. Sin embargo, el perímetro y la estructura tridimensional del asentamiento se ven alterados en las últimas décadas por obstáculos en forma de construcciones auxiliares. Su gran volumen y elevada altura llegan a impedir en ocasiones la visión de zonas enteras del núcleo. Aunque en 
muchos casos se sigue apreciando la forma originaria, en otros se produce una seria distorsión de las escenas tradicionales por parte de estos elementos periféricos. En concreto, se ha constatado la aparición de numerosas naves industriales agrícolas que representan una clara amenaza para la percepción del paisaje característico de Tierra de Campos. Estas construcciones de factura ingenieril suelen rechazar cualquier relación visual, ya sea volumétrica o material, con el tejido residencial formado mayoritariamente por conjuntos agregados de arquitectura popular.

Figura 8. Vía de acceso al núcleo de Aguilar de Campos, con presencia de naves agrícolas.

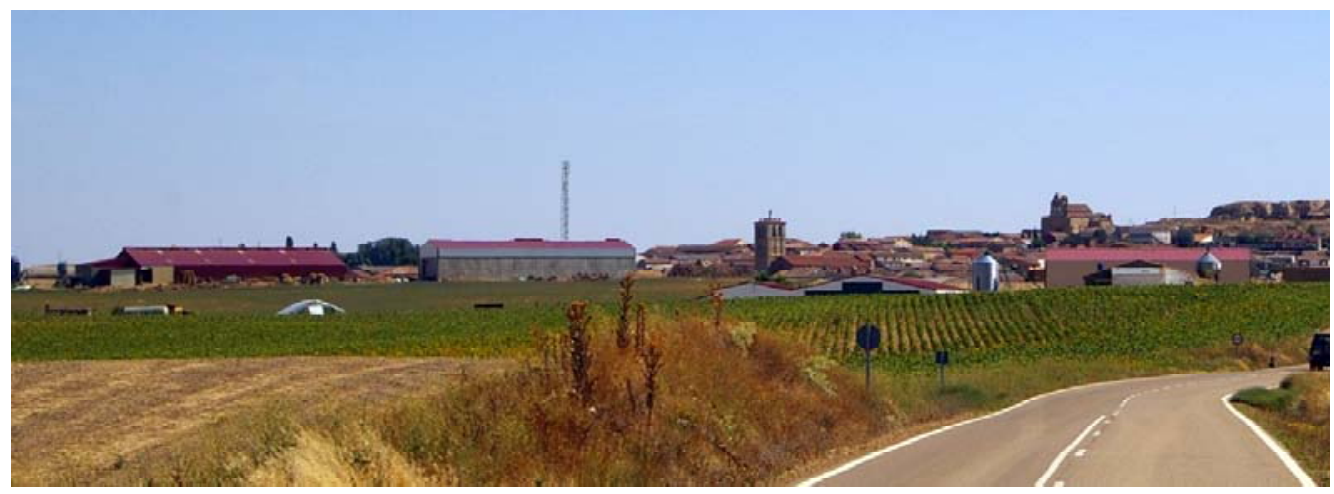

Fuente: Elaboración propia.

El desuso de los sistemas constructivos tradicionales es consecuencia de una menor dependencia del medio natural de una sociedad altamente industrializada, en comparación con las comunidades agrarias tradicionales. Tiene como efecto la desvinculación de los asentamientos del resto de elementos territoriales, debilitando su identidad cultural (Cortés Pedrosa, J., 2014). Las modernas técnicas constructivas se intentan justificar por una supuesta economía de medios, conseguida a través de la industrialización de elementos y el uso de materiales ligeros. Es el caso de los paneles prefabricados o la chapa corrugada. Sin embargo, la presencia explícita de estos materiales, con cualidades contrapuestas a los de la arquitectura tradicional de la zona, los hacen destacar negativamente dentro de la imagen física de los asentamientos. Las nuevas fachadas y cubiertas muestran acabados superficiales metálicos brillantes, lacados en colores primarios saturados, como el rojo y el verde, que contrastan fuertemente con los tenues tonos ocres y pardos de los materiales tradicionales, como la teja árabe o el tapial, generando discontinuidades en los conjuntos en que se inscriben. 
Figura 9. Nave agrícola en el núcleo de Bolaños de Campos.

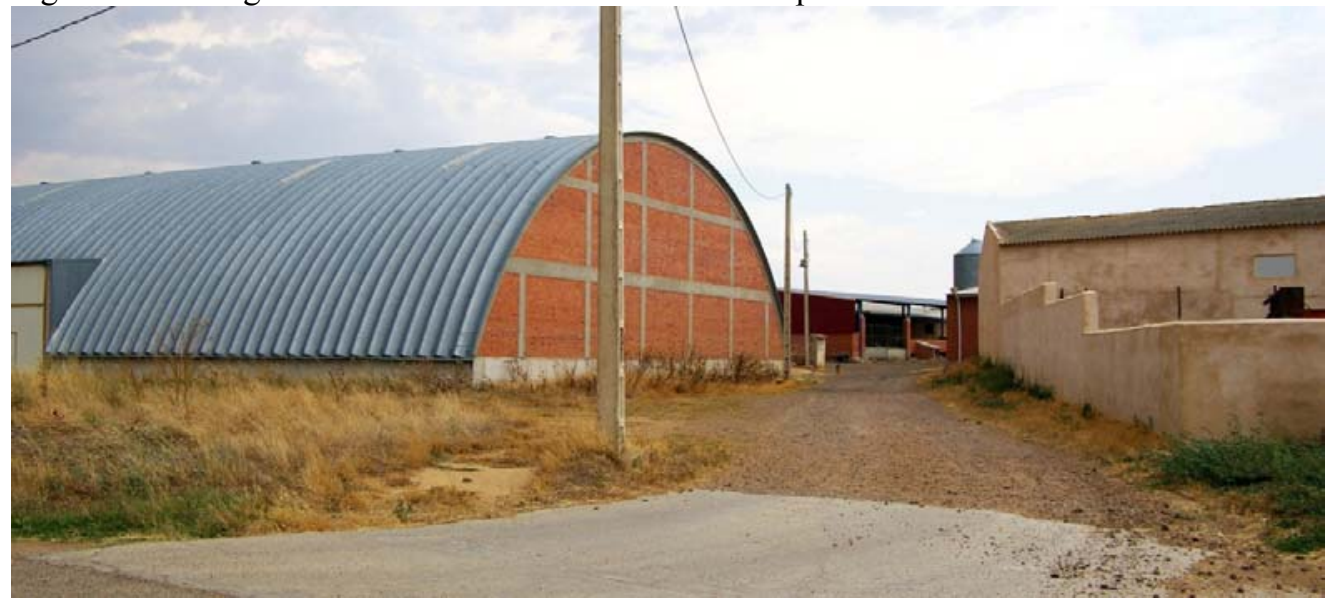

Fuente: Elaboración propia.

Otra situación frecuente es la transformación inadecuada del patrimonio de arquitectura popular, debido a los prejuicios existentes sobre su calidad constructiva o con el propósito de reducir la inversión en su mantenimiento. En las típicas casas con corral de la comarca se observan nuevos muros en bloque de hormigón o enfoscados de cemento, materiales que carecen de las ricas texturas y los delicados matices del tapial o el tradicional trullado, produciéndose un claro empobrecimiento estético.

Figura 10. Vista desde la entrada al núcleo de Tamariz de Campos.

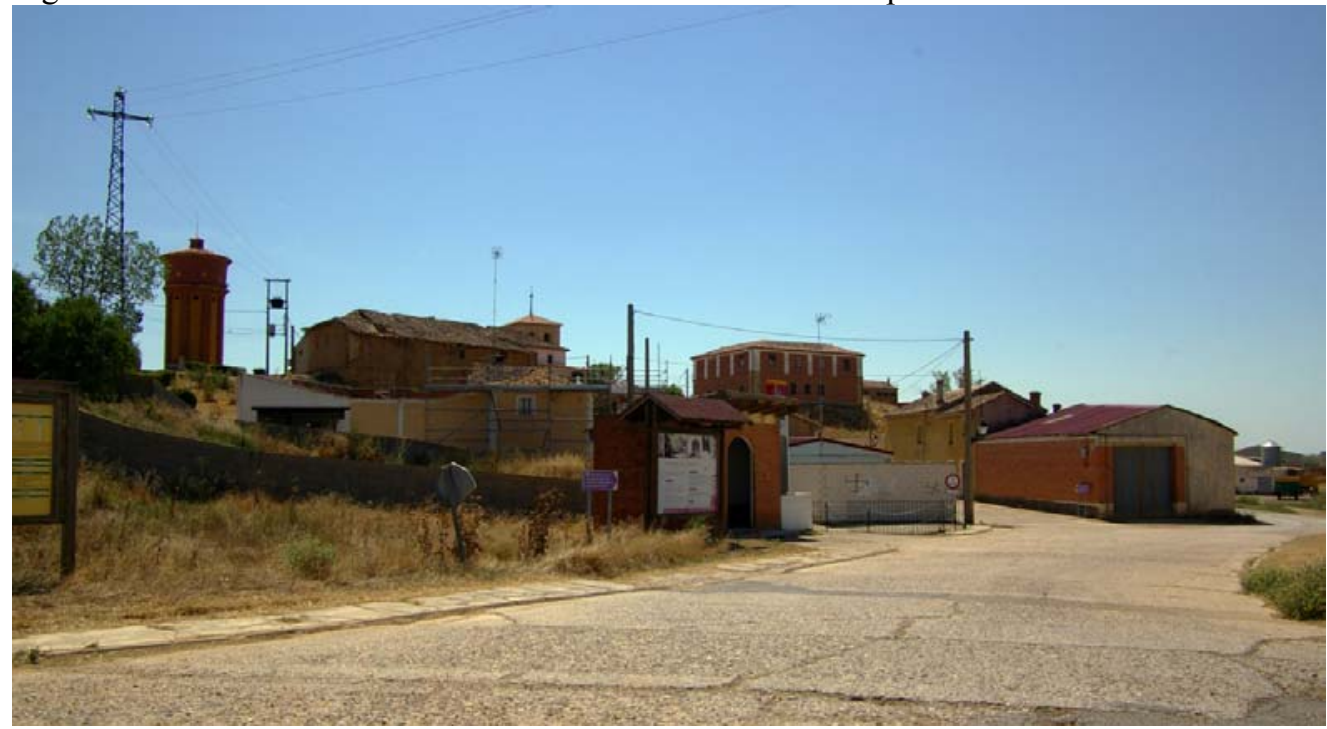

Fuente: Elaboración propia. 


\section{A MODO DE CONCLUSIÓN}

Algunos factores del medio físico, como el relieve suavemente ondulante y los suelos arcillosos, han permitido la práctica de una agricultura de secano en esta comarca desde hace más de dos milenios, favoreciendo una intensa presencia humana en el territorio. Los procesos culturales transformaron lentamente el soporte natural, destacando entre ellos las prácticas constructivas tradicionales, basadas en el empleo de los materiales térreos en forma de gruesos muros de carga. Esto generó, a su vez, unos asentamientos de gran unidad formal y material, que lograron una perfecta integración visual en su entorno. El paisaje cultural permitía visualizar un sutil e inteligente sistema de relaciones con el medio ecológico, basado en el aprovechamiento racional de los recursos naturales.

Pero el advenimiento de la era industrial desencadena unos procesos que transforman la realidad socioeconómica. La incapacidad de Tierra de Campos de adaptarse con éxito a las nuevas dinámicas globales, como la restructuración productiva, provoca cambios demográficos a partir de la mitad del siglo XX. Destaca la baja densidad de habitantes, que tiene efectos negativos sobre el patrimonio, como la falta de medios, humanos y económicos para la adecuada rehabilitación de la arquitectura popular. Se ha comprobado también que los núcleos de población sufren importantes alteraciones de su morfología tradicional por la aparición desordenada de nuevos desarrollos urbanísticos. Esto provoca un serio problema de degradación del paisaje, debido a la proliferación de edificaciones de tipo industrial, desligadas de la identidad territorial. Así pues, parece necesario modificar y adecuar las herramientas de planificación física y ordenación urbanística, para que contemplen esta situación y hagan frente al actual deterioro.

\section{BIBLIOGRAFÍA}

APARICIO GERVÁS, J. M. et alii (2005). Estudio, situación y análisis de la población inmigrante de "Tierra De Campos" (Zona norte de la provincia de Valladolid). Universidad de Valladolid, Valladolid.

BARAJA RODRÍGUEZ, E. (2012). La Tierra de Campos. En: MOLINERO, F. OJEDA, J. F. y TORT, J.: Los paisajes agrarios de España. Caracterización, evolución y tipificación. Ministerio de Medio Ambiente y Medio Rural y Marino, Madrid.

BARBA Y CASANOVAS, R. (1996). Paisaje. Entre el análisis del entorno y el diseño del espacio exterior. Revista Geometría, n. ${ }^{\circ} 21$; pp. 3-17.

BENITO MARTÍN, F. (1998). La arquitectura tradicional de Castilla y León. Junta de Castilla y León, Consejería de Medio Ambiente y Ordenación del Territorio, Valladolid.

BENITO MARTÍN, F. (2000). La formación de la ciudad medieval: La red urbana en Castilla y León. Secretariado de Publicaciones e Intercambio, Valladolid. 
CORTÉS PEDROSA, J. (2013). La arquitectura popular como modelo de edificación sostenible. El ejemplo de Tierra de Campos. Observatorio Medioambiental 2013, vol. 16; pp. 185-206, Universidad Complutense, Madrid.

CORTÉS PEDROSA, J. (2014). El espacio construido en Castilla y León: aspectos medioambientales. M+A. Revista Electrónic@ de Medio Ambiente 2014, vol. 15, $\mathrm{n}^{\mathrm{o}} 1$, pp. 1 - 21. Universidad Complutense, Madrid.

DIAZ PINEDA, F. (2003). Paisaje y Territorio. Colección Mediterráneo Económico n 4 : "Mediterráneo y Medio Ambiente".

FORMAN, R. T. T. y Godron, M. (1986). Landscape Ecology. John Wiley \& Sons, New York, EE.UU.

FORMAN, R. T. T. (1996). Land mosaics: the ecology of landscapes and regions. Cambridge University Press, Cambridge, Massachusetts, EE.UU.

FLORES LÓPEZ, C. (1974). Arquitectura popular española. Volumen $1^{\circ}$. Aguilar, Madrid, 1976.

GARCÍA MERINO, L. V. (1988). Análisis del Medio Físico de Valladolid. Delimitación de unidades y estructura territorial. Junta de Castilla y León, Valladolid.

GONZÁLEZ GARRIDO, J. (1941). La Tierra de Campos, región natural. Horizontes de Castilla, Valladolid.

HERNÁNDEZ GARCÍA, R. (2011). Vacceos ¿identidad de pasado o de futuro? El Futuro del Pasado, no 2, 2011; pp. 353-369.

INSTITUTO NACIONAL DE ESTADÍSTICA (2013). Padrón municipal.

MARGALEF, R. (1980). La biosfera entre la termodinámica y el juego. Omega, Barcelona.

MARTÍNEZ DE PISÓN, E. et alii (2004. La conservación del paisaje. Fundación biodiversidad, Sevilla.

MARTÍNEZ FERNÁNDEZ, L. C. y DELGADO URRECHO, J. M. (2013). Población, administración y territorio en Castilla y León: desequilibrios y desafíos del modelo de poblamiento. Ería, $\mathrm{n}^{\circ}$ 90; pp. 5-30.

GARCÍA MERINO, L. V. (1998). Análisis del Medio Físico de Valladolid. Delimitación de unidades y estructura territorial. Junta de Castilla y León, Valladolid.

MOYA, L. et alii (2014). Estudio de la tipología arquitectónica y urbanística en el medio rural de la provincia de Valladolid. ETS de Arquitectura de Valladolid, Grupo TIERRA.

PLAZA GUTIÉRREZ, J. I. (2010). Ordenación y desarrollo del territorio en Castilla y León. Análisis y valoraciones. Cuadernos Geograficos, 47 (2010-2); pp. 523552.

RAFFESTIN, C. (1980). Pour une géographie du pouvoir. LITEC, Paris. [Traducción propia]

ROLDÁN MORALES, F. P. (1983). Palomares de barro de Tierra de Campos. Caja de Ahorros Provincial de Valladolid.

ROLDÁN MORALES, F. P. (1996). Arquitectura popular de la provincia de Valladolid. Diputación Provincial de Valladolid. 
SAUER, C. O. (1925). The Morphology of Landscape. University of California Publications in Geography 2(2), pp. 19-53. [Traducción propia]

ROGER, A. (2007). Breve tratado del paisaje. Biblioteca Nueva, Madrid.

SÁINZ GUERRA, J.L. et alii (2012). Edificios y conjuntos de la arquitectura popular en Castilla y León. Edición electrónica: Consejería de Cultura y Turismo, Junta de Castilla y León.

SANTOS Y GANGES, L. Y PEIRET I CARRERA, A. (2001).: Articulación regional y comarcas en Castilla y León: las directrices de ordenación del territorio. Boletín de la A.G.E. no 32; pp. 177-190.

SOTELO NAVALPOTRO, J. A. (1991). Paisaje, semiología y análisis geográfico. Anales de Geografía de la Universidad Complutense, nº 11, pp. 11-23.

VACA LORENZO, A. (1977). La estructura socioeconómica de la Tierra de Campos a mediados del siglo XIV. Publicaciones de la Institución Tello Téllez de Meneses, $n^{\circ} .39$.

VACA LORENZO, A. (1992). La Tierra de Campos y sus bases ecológicas en el siglo XIV. Studia Histórica. Historia Medieval, no 10, pp. 149-186, Ediciones Universidad de Salamanca. 